\title{
Review of food bolus management
}

\author{
Hin Hin Ko MD FRCPC, Robert Enns MD FRCPC
}

\begin{abstract}
$\mathrm{F}$ gists. Fortunately, the majority of ingested foreign objects and food boluses will pass spontaneously. However, $10 \%$ to $20 \%$ may become lodged in the esophagus or other locations in the gastrointestinal tract and may require nonoperative intervention, and $1 \%$ or less will require surgery (1-4). A recent study (5) reported an estimated annual incidence rate of food impactions of 13 episodes per 100,000 in a health maintenance organization population. The overall mortality rate is unknown, but deaths have been rarely reported $(6,7)$. The purpose of the present review is to focus on esophageal food bolus impaction - its pathophysiology, clinical presentations, diagnosis and management.
\end{abstract} Toreign object ingestion and esophageal food bolus impactions are common problems faced by gastroenterolo-

\section{PATHOPHYSIOLOGY}

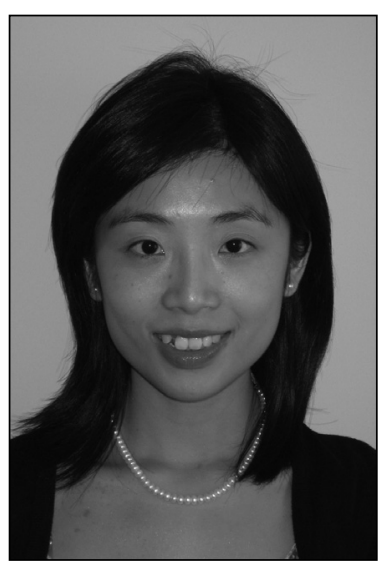

Dr Hin Hin Ko throat pain (9). Odynophagia may occur as a result of distension of the esophagus by food bolus, but it is also a marker for esophageal injuries such as laceration, abrasion or perforation. Retching and emesis are also very common, and patients sometimes try to self-induce vomiting in an attempt to dislodge the object. Patients with a high-grade esophageal obstruction may experience hypersalivation and may be unable to swallow any liquids, including their own saliva.

Respiratory symptoms may result from compression of the trachea by a large food bolus impaction (rare), from aspiration of saliva or food (more common), or complete airway obstruction. Common respiratory symptoms include stridor, coughing, wheezing or choking.

\section{DIAGNOSIS}

Food impaction usually occurs as a result of two factors: the state of the esophagus, and the nature of the food that has been swallowed. Previous studies (5), revealed that food bolus impactions have underlying esophageal pathology in $88 \%$ to $97 \%$ of adult patients evaluated. Benign esophageal stenoses caused by Schatzki (B) rings or by peptic strictures are the most common causes, followed by webs, extrinsic compression, surgical anastomosis, esophagitis and motor disorders such as achalasia. Surprisingly, food bolus impaction is an uncommon presentation for esophageal carcinoma. One other emerging condition that is especially important to recognize is eosinophilic esophagitis. It is commonly found in younger patients presenting with food dysphagia and impaction. A recent study (8) showed that up to $54 \%$ of adults who presented with esophageal food impactions over a three-year period had histological evidence of eosinophilic esophagitis. When these underlying esophageal conditions are present and, if food, especially meat, is poorly chewed and swallowed precipitously, acute food impaction can occur.

\section{CLINICAL PRESENTATIONS}

Food bolus impaction usually presents acutely, especially in adults who have had a clear history of ingestion. Impaction at the upper esophageal sphincter is generally easily localized by the patient. However, impaction at sites lower in the esophagus may cause symptoms such as diffuse chest pain or pressure, dysphagia, odynophagia, a sensation of choking, and neck or
Diagnosis of food bolus impaction is rarely a problem because most patients are able to relate the type of food ingested and the approximate time symptoms began. The goal of the initial patient assessment is to determine the stability of the patient, the type of food ingested and time since ingestion, the presence of any complications and the presence of any underlying esophageal conditions or other medical comorbidities.

A careful history should inquire of any previous history of dysphagia, food impaction, gastroesophageal reflux disease, known structural esophageal abnormalities and the contents of meals. In general, the patient's localization of food impaction is often unreliable and esophageal dysphagia is more likely to be referred proximally, rather than distally, from the site of obstruction $(10,11)$.

The physical examination should focus on the assessment of the patient's stability and any complications from the food bolus impaction. Ventilation, airway compromise and the risk of aspiration should first be assessed. Signs suggestive of perforation include fever, tachycardia, subcutaneous crepitus and swelling of the neck or chest. Esophageal perforation may require surgical intervention, and consultations should not be delayed.

Radiographic evaluation is useful to detect any associated complications, such as free mediastinal air from perforation; however, food boluses, including fish or chicken bones, are radiolucent and not readily visualized on plain radiographs. A contrast examination should not be performed because of the risk of aspiration. 


\section{MANAGEMENT}

\section{Endoscopy}

Flexible endoscopy is highly sensitive in detecting food boluses and has the advantage of delivering immediate therapy in most cases. Furthermore, endoscopy can reveal the underlying esophageal pathology leading to the impaction and any associated mucosal damage. Successful management of food bolus impaction is influenced by many factors including the experience and technical skills of the endoscopist, as well as the availability of necessary equipment and accessories. As stated above, flexible endoscopy is the preferred treatment modality for most cases of esophageal food impaction. Furthermore, endoscopic diagnosis and treatment of the underlying pathology can be performed at the same setting. The goal of therapy is to clear the esophagus by either extracting the bolus or moving it into the stomach to prevent any complications.

Timing of endoscopy: The timing of endoscopy is dictated by the perceived risks of aspiration and/or perforation. Urgent intervention is indicated when patients are in severe distress. In patients unable to ingest their own secretions, the use of a suction catheter or Yankauer sucker, which can be held by the patient while in a sitting position, is useful while waiting for endoscopic intervention.

Patients who have no evidence of high-grade obstruction and who are in no acute distress can be handled less urgently because spontaneous passage of food boluses may occur and endoscopy can be postponed until a more convenient time (1-4). However, endoscopic intervention should not be postponed beyond $24 \mathrm{~h}$ from presentation because of the increased risk of complications $(12,13)$. Early removal of the food bolus may minimize the amount of local pressure-induced mucosal damage in the esophagus. Smith and Wong (14) even suggested performing nonurgent endoscopy within the first $6 \mathrm{~h}$ to $12 \mathrm{~h}$ to increase the likelihood of removing a meat bolus in one piece, before it has a chance to soften. The actual timing of the endoscopy is often delayed a few hours in the early morning until a more convenient endoscopy time (usually 07:00) can be arranged. This seems to be a reasonable approach and, providing that the time for endoscopy fits into the above stated time period, is likely a safe approach.

Equipment: Readily available equipment should include appropriately sized endoscopes, overtubes, accessories such as polypectomy snares, rat-tooth and alligator forceps, a Roth retrieval net, Dormia basket, and Magill or Kelly grasping forceps (14).

Airway protection: During extraction, it is essential to protect the patient's airway from inadvertent aspiration of saliva and retained esophageal food contents and the food itself. Ideally, assistants should be available to provide oral suctioning and monitor the patient's vital signs. Removal of foreign bodies can be the easiest (requiring simple sedation and insufflation of the esophagus alone) and, at times, the most difficult of procedures. Endotracheal intubation may be needed if the patient is uncooperative or at a high-risk of aspiration. The esophagus should be intubated under direct visualization and any retained secretions in the esophagus should be suctioned to improve visualization and reduce the aspiration risk. Once the food bolus is identified, the endoscopist should decide which technique to use to clear the esophagus and what accessory equipment is needed.

Removal techniques: Pushing: There are many reported endoscopic techniques to facilitate removal of food boluses. Considerable disagreement exists concerning whether a bolus should be extracted or pushed into the stomach. Bolus extraction is widely advocated (15-17) and, historically, pushing has been avoided because of the concern for associated distal obstructing lesions, or strictures and increased risk of perforation. However, several authors $(5,18-20)$ have recently advocated using the push technique to guide the esophageal food bolus into the stomach. The push technique is advocated in certain situations such as in patients without a history of dysphagia or those in whom the food bolus is soft or composed of many small fragments, making extraction difficult $(1,4,21)$. Vicari et al (20) reported a $97 \%$ success rate using the push technique for acute esophageal food impaction.

The push technique requires experience and good judgement. It is generally safe if the endoscope, with insufflation and distension of the esophageal lumen, can be steered successfully beyond the bolus into the stomach. The endoscope can then be pulled back to a position just above the bolus. With the tip of the endoscope, gentle pressure is applied to the bolus, carefully guiding it into the stomach. It is important to push from the right side of the food bolus because in the setting of a hiatus hernia, the gastroesophageal junction has a left turn; pressure from the right then allows a bolus to pass distally (22). A Savary-Gilliard (Cook Medical, USA) dilator push method has also been reported, in which a wire is gently inserted past a food bolus into the stomach under direct visualization. One or two small diameter Savary-Gilliard dilators (28 Fr or 32 Fr catheter) are then passed with subsequent endoscopic examination of the esophagus (18). A retrospective review (18) showed that the pushing technique was successful in 48 patients using the endoscope alone and in 20 patients by Savary-Gilliard dilator push method; however, this method is not usually required in most patients.

In instances when the bolus cannot be passed with the endoscope, the push technique may still be attempted; however, the procedure should be stopped if any significant resistance is encountered. Blindly pushing the impacted food bolus with the endoscope is not recommended due to the high incidence of underlying esophageal pathology (4).

When gentle pushing is unsuccessful, one can use forceps, snares or baskets (biliary or Roth-type) to attempt breaking the food bolus into smaller pieces for extraction.

Extraction: Extraction is the preferred technique when the food bolus is large, firm, contains bones or sharp edges, or the patient is known to have significant stricture. Using a variety of snares, baskets, graspers or forceps, the food bolus can usually be removed in toto in a piecemeal fashion. When using a polypectomy snare, it should be pulled snug against the tip of the endoscope once the food bolus has been successfully ensnared (23). The Roth retrieval net has the added benefit of encompassing the food bolus completely within the net, allowing a firmer grasp and minimizing the risk of dislodging any food fragments in the hypopharynx and airway during the retrieval process. This technique may also result in fewer repeated passes of the endoscope and thus, results in a significantly shorter duration of endoscopy and sedation compared with the use of other instruments (24).

If the food bolus is impacted tightly or lodged proximally near the cricopharynx, it can be difficult to grasp with a snare or Roth net. In those instances, many endoscopists prefer to use an endoscopic suction technique. A transparent endoscopic vacuum cap, such as those found in variceal ligation and endoscopic mucosal resection kits, is fit over the endoscope. The 
continuous suctioning then creates a vacuum between the tip of the endoscope and the foreign body, thereby pulling the food bolus against the cap $(25,26)$. One problem with this technique can be a failure to keep the bolus secured simply by suction, despite using greater suction forces. Patel et al (27) reported modifying the suction cap technique by using a Dormia basket. The basket is advanced into the endoscopic channel through the suction cap. The food bolus is then grasped with the basket, then the endoscope with the suction cap is slowly advanced toward the impaction while the Dormia basket grasping the bolus is carefully pulled toward the endoscope. Once the bolus is inside the suction cap, full suction is applied and the impacted food is extracted by means of this combination method (27).

In situations when the bolus has become softer and fragmented, and repeated intubation seems unavoidable, occasional use of an overtube is helpful. After lubrication of the internal and external surfaces, a 44 Fr catheter Maloney dilator (Medovation, USA) is passed through the tube, and the entire unit is guided into the esophagus and the dilator is subsequently removed (28). Use of an overtube offers airway protection and allows for multiple passes of the endoscope during retrieval (29). Younger physicians and trainees may have very little experience with this device because it is required only in very selected cases.

\section{Medical therapy}

A few pharmacological agents may be tried on admission to the emergency room or while preparing for endoscopy. Glucagon is the mainstay pharmacological treatment. It is an endogenous polypeptide secreted from alpha cells in the islets of Langerhans. At pharmacological doses, it relaxes the esophageal smooth muscle and the lower esophageal sphincter, promoting the spontaneous passage of an impacted food bolus (30). Glucagon seems to have very little effect on the motility of the proximal esophagus (31). In a person with normal esophageal anatomy, the resting lower esophageal sphincter pressure decreases by up to $60 \%$ after intravenous glucagon administration (30). In one study (30), glucagon given at the time of endoscopy facilitated clearing of the food bolus, probably secondary to its smooth muscle relaxation effect. The success rate for the use of glucagon is reportedly between $12 \%$ and $50 \%(8,32-34)$. However, glucagon is much less effective in patients with structural abnormalities, such as strictures or rings.

Contraindications for the use of glucagon include insulinoma, pheochromocytoma, Zollinger-Ellison syndrome or drug sensitivity. The dose of glucagon normally used is $1 \mathrm{mg}$ to $2 \mathrm{mg}$ intravenously in adults and $0.02 \mathrm{mg} / \mathrm{kg}$ to $0.03 \mathrm{mg} / \mathrm{kg}$ in children weighing less than $20 \mathrm{~kg}$, with a maximum dose of $0.5 \mathrm{mg}$. Repeat dosing at $5 \mathrm{~min}$ to $10 \mathrm{~min}$ is reasonable if needed (35); however, if the first two doses are ineffective, further doses are

\section{REFERENCES}

1. Webb WA. Management of foreign bodies of the upper gastrointestinal tract. Gastroenterology 1988;1:204-16.

2. Henderson CT, Engel J, Schlesinger P. Foreign body ingestion: Review and suggested guidelines for management. Endoscopy 1987;19:68-71.

3. Nandi P, Ong GB. Foreign body in the esophagus: Review of 2394 cases. Br J Surg 1979;65:5-9.

4. Vizcarrondo FJ, Brady PG, Nord HJ. Foreign bodies of the upper gastrointestinal tract. Gastrointest Endosc 1983;20:208-10.

5. Longstreth GF, Longstreth KJ, Yao JF. Esophageal food not indicated $(9,35)$. Glucagon is not a medication free of side effects. It is commonly associated with nausea or vomiting that could be harmful in the presence of a severe impaction and poses a theoretical risk of perforation and aspiration (36).

Several gas-forming agents have also been used to treat acute esophageal food impactions. These agents release carbon-dioxide in the esophagus, which raises the intraluminal pressure against a closed upper esophageal sphincter, forcing the bolus into the stomach. Examples of these agents include simethicone, carbonated beverages and a cocktail of tartaric acid and bicarbonate. Success has been reported $(37,38)$, however, complications such as esophageal perforation have also been reported (28).

Use of proteolytic enzymes such as papain or chymotrypsin in an attempt to dissolve food boluses have fallen out of favour because of potentially severe complications including esophageal erosion, necrosis and perforation, as well as severe pulmonary complications such as hemorrhagic pulmonary edema if the solution is aspirated $(1,39,40)$. Proteolytic enzyme preparations have no place in the current management of food bolus impaction.

\section{Management after food bolus clearance}

After the food bolus has been removed, endoscopic assessment should be completed. At times, this may be necessary to complete on a different date in a more elective manner, particularly if the patient has been unstable. Patients who have food impactions commonly are found to have underlying esophageal pathologies such as a peptic stricture or Schatzki ring. A decision would have to be made as to whether to dilate the associated stenosis at the same or different sessions. If there is minimal mucosal damage and the bolus has been present for a short period of time, dilation can be performed at the same setting $(17,22)$. However, if there is significant inflammation or mucosal damage, the patient can be placed on acid suppressants and brought back for dilation at a later date.

In patients who present with a history of esophageal complaints and have an endoscopic appearance suspicious of eosinophilic esophagitis (eg, linear mucosal furrows, mucosal rings, small-calibre esophagus or characteristic mucosal white exudates), dilation should likely be avoided because it may be associated with a higher incidence of complication and greater likelihood of recurrence $(41,42)$. Unless contraindicated, biopsies of the proximal and distal esophagus should be performed to confirm the diagnosis.

\section{SUMMARY}

Esophageal food impaction is a common emergency faced by gastroenterologists. It is important to know what techniques and instruments are indicated for different situations. Endoscopic treatment is a reliable and safe procedure in skilled, expert hands with a high success rate and low morbidity and mortality.

impaction: Epidemiology and therapy. A retrospective, observational study. Gastrointest Endosc 2001;53:193-8.

6. Simic MA, Budakov BM. Fatal upper esophageal hemorrhage caused by a previously ingested chicken bone: Case report. Am J Forensic Med Pathol 1988;19:166-8.

7. Bennett DR, Baird CJ, Chan KM, et al. Zinc toxicity following massive coin ingestion. Am J Forensic Med Pathol 1997;18:148-53.

8. Desai TK, Stecevic V, Chang CH, et al. Association of eosinophilic inflammation with esophageal food impaction in adults. Gastrointest Endosc 2005;61:795-801. 
9. Taylor RB: Esophageal foreign bodies. Emerg Med Clin North Am 1987;5:301-11.

10. Johnston B, Castell D. Symptom overview and quality of life. In: Castell D, Richter J, ed. The Esophagus. Philadelphia: Lippincott Williams \& Wilkins;2004:37-46.

11. Wilcox, CM, Alexander LN, Clark WS. Localization of an obstructing esophageal lesion. Is the patient accurate? Dig Dis Sci 1995;40:2192-6.

12. Eisen GM, Baron TH, Dominitz JA, et al. Guideline for the management of ingested foreign bodies. Gastrointest Endosc 2002;55:802-6.

13. Chaves DM, Ishioka S, Felix VN, et al. Removal of a foreign body from the upper gastrointestinal tract with a flexible endoscope: A prospective study. Endoscopy 2004;36:887-92.

14. Smith MT, Wong RKH. Esophageal foreign bodies: Types and techniques for removal. Curr Treat Options Gastroenterol 2006;9:75-84

15. Shaffer RD, Klug T. A comparative study of techniques for esophageal foreign body removal with special emphasis on meat bolus obstruction. Wis Med J 1981;80:33-6.

16. Berggreen PJ, Harrison E, Sanowski RA, et al. Techniques and complications of esophageal foreign body extraction in children and adults. Gastrointest Endosc 1993;39:626-30.

17. Yeaton P, Peura DA. Foreign bodies. In: Castell DO, Richter JE, eds. The Esophagus, 3rd edn. Philadelphia: Lippincott Williams \& Wilkins, 1999:335-48.

18. Weinstock LB, Shatz BA, Thyssen SE. Esophageal food bolus obstruction: Evaluation of extraction and modified push techniques in 75 cases. Endoscopy 1999;31:421-5.

19. Kozarek R, Ball T, Belic L, et al. Food impaction at a regional referral centre: Should we push? Pull? Or poke? Gastrointest Endosc 1999;49:113 (Abst).

20. Vicari JJ, Johnason JF, Frakes JT. Outcomes of acute esophageal food impaction: Success of the push technique. Gastrointest Endosc 2001;53:178-81.

21. Ginsberg G. Management of ingested foreign objects and food bolus impactions. Gastrointest Endosc 1995;41:33-8.

22. Webb WA. Management of foreign bodies of the upper gastrointestinal tract: Update. Gastrointest Endosc 1995;41:39-51.

23. Webb WW, Taylor MB. Foreign bodies of the upper gastrointestinal tract. In: Taylor MB, ed. Gastrointestinal Emergencies, 2nd edn. Baltimore: Williams \& Wilkins, 1997:3-19.

24. Neustater B, Barkin JS. Extraction of an esophageal food impaction with a Roth retrieval net. Gastrointest Endosc 1996;43:66-7.

25. Pezzi JS, Shiau YF. A method for removing meat impactions from the esophagus. Gastrointest Endosc 1994;40:634-6.
26. Nijhawan S, Rai RR, Nepalia S, et al. Suction retrieval of esophageal foreign bodies. Endoscopy 1998;30:S59.

27. Patel NC, Fry LC, Monkemuller KE. Modified suction cap technique for endoscopic management of esophageal food impaction. Endoscopy 2003;35:548.

28. Smith MT, Wong RKH. Foreign Bodies. Gastrointest Endosc Clin N Am 2007;17:361-82.

29. Guideline for the management of ingested foreign bodies. American Society for Gastrointestinal Endoscopy. Gastrointest Endosc 1995;42:622-5.

30. Alaradi O, Bartholomew M, Barkin JS. Upper endoscopy and glucagon: A new technique in the management of acute esophageal food impaction. Am J Gastroenterol 2001;96:912-3.

31. Colon V, Grade A, Pulliam G, et al. Effect of doses of glucagon used to treat food impaction on esophageal motor function of normal subjects. Dysphagia 1999;14:27-30.

32. Blair SR, Graeber GM, Cruzzavala JL, et al. Current management of esophageal impactions. Chest 1993;104:1205-9.

33. Brady PG. Esophageal foreign bodies. Gastroenterol Clin North Am 1991;20:691-70.

34. Giordano A, Adams G, Boies L, et al. Current management of esophageal foreign bodies. Arch Otolaryngol 1981;107:249-51.

35. Lyons MF, Tsuchida AM. Foreign bodies of the gastrointestinal tract. Med Clin North Am 1993;77:1102-14.

36. Al-Haddad M, Ward EM, Scolapio JS, et al. Glucagon for the relief of esophageal food impaction: Does it really work? Dig Dis Sci 2006;51:1930-3.

37. Karanjia ND, Rees M. The use of Coca-Cola in the management of bolus obstruction in benign esophageal strictures. Ann R Coll Surg Engl 1993;75:94-5.

38. Lee J, Anderson R. Best evidence topic report. Effervescent agents for esophageal food bolus impaction. Emerg Med J 2005;22:123-4.

39. Hall ML, Huseby JS. Hemorrhagic pulmonary edema associated with meat tenderizer for esophageal meat impaction. Chest 1988;94:640-2.

40. Straumann A, Spichtin HP, Grize L, et al. Natural history of primary eosinophilic esophagitis: A follow-up of 30 adult patients for up to 11.5 years. Gastroenterology 2003;125:1660-9.

41. Kaplan M, Mutlu EA, Jakate S, et al. Endoscopy in eosinophilic esophagitis: "Feline" esophagus and perforation risk. Clin Gastroenterol Hepatol 2003;1:433:7.

42. Vasilopoulos S, Shaker R. Defiant dysphagia: A small-calibre esophagus and refractory benign esophageal strictures. Curr Gastroenterol Rep 2001;3:225-30. 


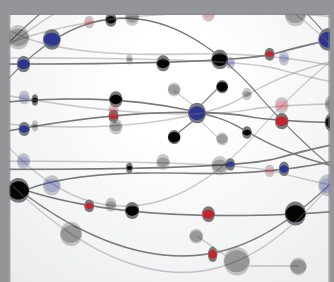

The Scientific World Journal
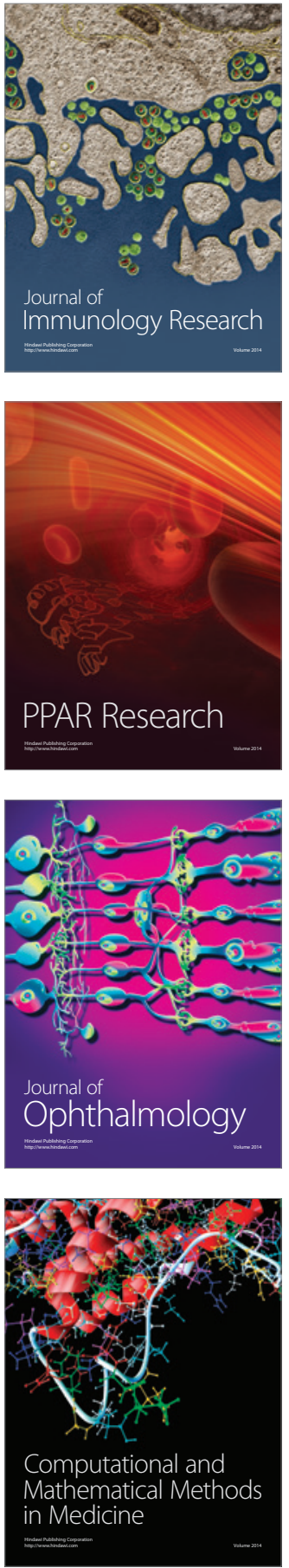

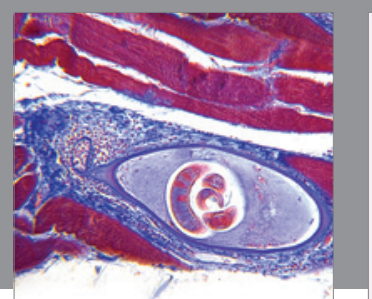

Gastroenterology Research and Practice

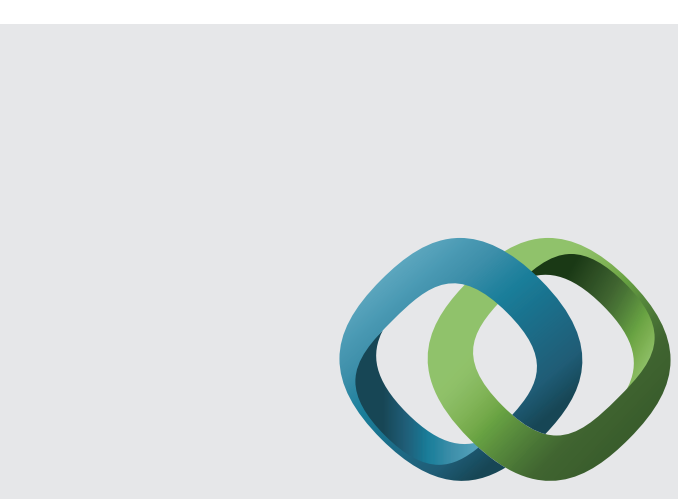

\section{Hindawi}

Submit your manuscripts at

http://www.hindawi.com
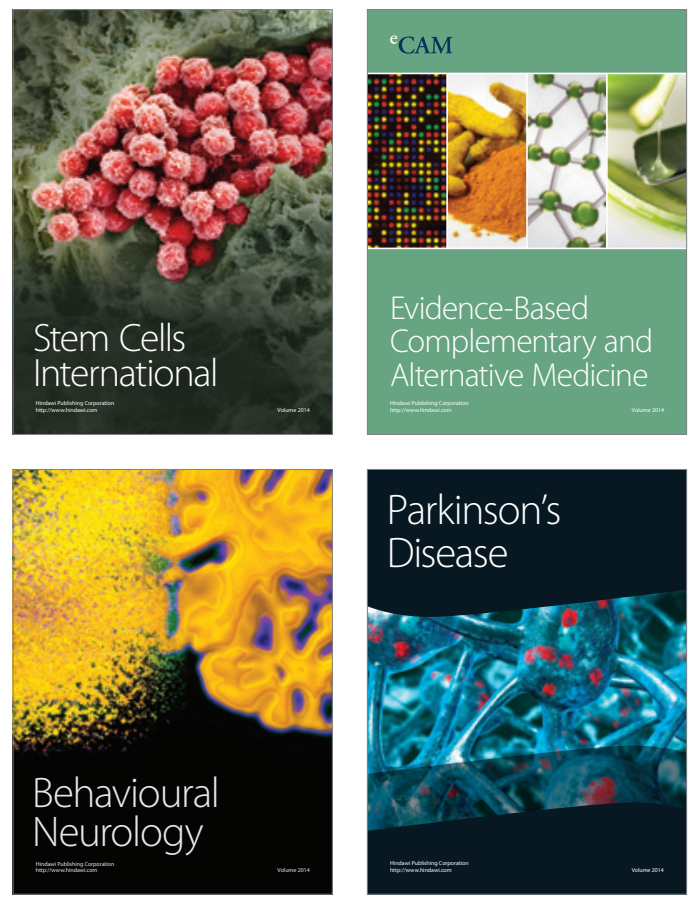
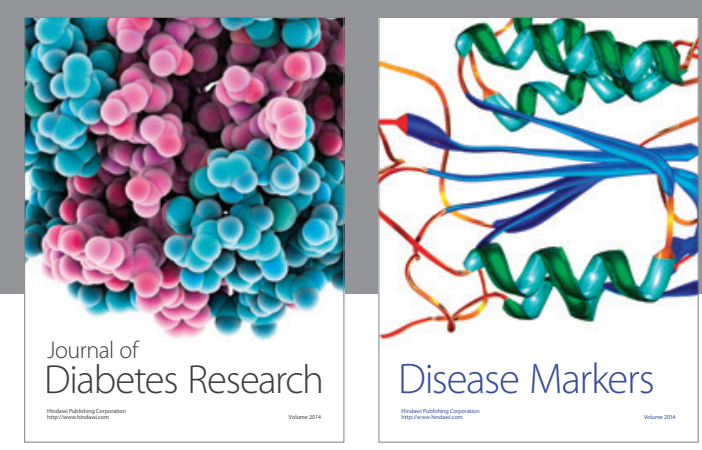

Disease Markers
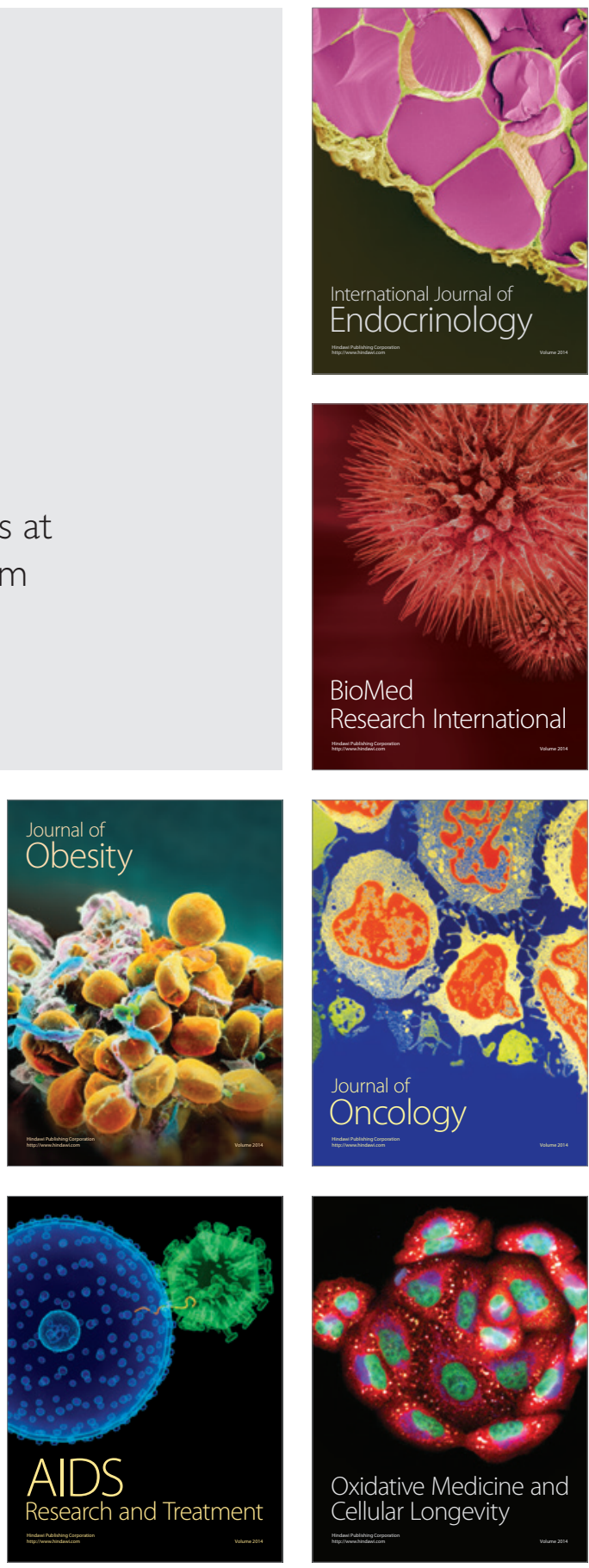